\title{
Biomechanical effects of hybrid stabilization on the risk of proximal adjacent-segment degeneration following lumbar spinal fusion using an interspinous device or a pedicle screw-based dynamic fixator
}

\author{
Chang-Hyun Lee, MD, MSc, ${ }^{1}$ Young Eun Kim, PhD, ${ }^{2}$ Hak Joong Lee, MSc, ${ }^{2}$ \\ Dong Gyu Kim, MD, PhD, ${ }^{3}$ and Chi Heon Kim, MD, PhD ${ }^{3}$

\begin{abstract}
1'Department of Neurosurgery, Ilsan Paik Hospital, Inje University College of Medicine, Goyang-si, Gyeonggi-do; 2Department of Mechanical Engineering, Dankook University, Yongin-si, Gyeonggi-do; and ${ }^{3}$ Department of Neurosurgery, Seoul National University Hospital, Seoul National University College of Medicine, Seoul, Republic of Korea
\end{abstract}

\begin{abstract}
OBJECTIVE Pedicle screw-rod-based hybrid stabilization (PH) and interspinous device-based hybrid stabilization (IH) have been proposed to prevent adjacent-segment degeneration (ASD) and their effectiveness has been reported. However, a comparative study based on sound biomechanical proof has not yet been reported. The aim of this study was to compare the biomechanical effects of $\mathrm{IH}$ and $\mathrm{PH}$ on the transition and adjacent segments.
\end{abstract}

METHODS A validated finite element model of the normal lumbosacral spine was used. Based on the normal model, a rigid fusion model was immobilized at the L4-5 level by a rigid fixator. The DIAM or NFlex model was added on the L3-4 segment of the fusion model to construct the $\mathrm{IH}$ and $\mathrm{PH}$ models, respectively. The developed models simulated 4 different loading directions using the hybrid loading protocol.

RESULTS Compared with the intact case, fusion on L4-5 produced $18.8 \%, 9.3 \%, 11.7 \%$, and $13.7 \%$ increments in motion at L3-4 under flexion, extension, lateral bending, and axial rotation, respectively. Additional instrumentation at L3-4 (transition segment) in hybrid models reduced motion changes at this level. The IH model showed $8.4 \%,-33.9 \%$, $6.9 \%$, and $2.0 \%$ change in motion at the segment, whereas the $\mathrm{PH}$ model showed $-30.4 \%,-26.7 \%,-23.0 \%$, and $12.9 \%$. At L2-3 (adjacent segment), the PH model showed $14.3 \%, 3.4 \%, 15.0 \%$, and $0.8 \%$ of motion increment compared with the motion in the IH model. Both hybrid models showed decreased intradiscal pressure (IDP) at the transition segment compared with the fusion model, but the pressure at L2-3 (adjacent segment) increased in all loading directions except under extension.

CONCLUSIONS Both $\mathrm{IH}$ and PH models limited excessive motion and IDP at the transition segment compared with the fusion model. At the segment adjacent to the transition level, $\mathrm{PH}$ induced higher stress than $\mathrm{IH}$ model. Such differences may eventually influence the likelihood of ASD.

https://thejns.org/doi/abs/10.3171/2017.3.SPINE161169

KEY WORDS hybrid stabilization; lumbar; finite element analysis; adjacent segment; interspinous device; pedicle screw; dynamic fixator

$\mathrm{O}$ VER the past several decades, lumbar spinal fusion with instrumentation has been commonly used in the surgical treatment of symptomatic lumbar degenerative diseases. Technological advances have resulted in increased fusion rates. However, achievement of fusion may have long-term effects on the adjacent motion segments, and adjacent-segment degeneration (ASD) has been reported to have a prevalence of more than $30 \% .^{4,9,21}$ This phenomenon is considered to be caused by the altered biomechanics of the fused spine, wherein abnormal forces acting upon the intervertebral discs and facet joints adjacent to the fused segment precipitate the accelerated failure of these stabilizing elements. ${ }^{2,5,16,22}$ Although the assumption of a fusion-related disease and the actual rate of ASD are debatable, it is often believed that the development of ASD is related to adaptive hypermobility in segments adjacent

ABBREVIATIONS ASD = adjacent-segment degeneration; FE = finite element; IDP = intradiscal pressure; $I H$ = interspinous device-based hybrid stabilization; ISD = interspinous device; $P D S$ = pedicle screw-based dynamic stabilization; $\mathrm{PH}=$ pedicle screw-rod-based hybrid stabilization; $\mathrm{ROM}=$ range of motion.

SUBMITTED October 4, 2016. ACCEPTED March 16, 2017.

INCLUDE WHEN CITING Published online September 22, 2017; DOI: 10.3171/2017.3.SPINE161169. 
to the instrumented fusion. . $^{15,27,37}$ This hypermobility or instability is usually observed rostral to a fused segment, and clinical observations have indicated that the level proximal to the fusion is more likely to undergo degenerative changes than the level distal to the fusion..$^{27,28}$

Based on this evidence for ASD, the concept of hybrid stabilization comprising rigid fixation and dynamic stabilization has emerged. ${ }^{2}$ Hybrid stabilization is generally classified into interspinous device (ISD)-based hybrid stabilization (IH; interspinous process stabilizer with conventional fusion) and pedicle screw-rod-based hybrid stabilization (PH; pedicle screw-rod construct with flexible rod at the adjacent upper segment). Several retrospective clinical studies ${ }^{1,20,25}$ have demonstrated the effectiveness of IH and PH in preventing ASD. ${ }^{1,11,20,23,25}$ However, a welldesigned prospective study based on sound biomechanical proof is lacking. Moreover, a biomechanical comparison between $\mathrm{IH}$ and $\mathrm{PH}$ under the same loading condition has not been reported until now.

Therefore, this study aimed to investigate the biomechanical effects of hybrid stabilization using an ISD or pedicle screw-based dynamic stabilization (PDS) system at the transition and adjacent segments after single-level lumbar fusion using a validated finite element (FE) model.

\section{Methods}

\section{Rigid Fusion Model}

A previously developed and validated lumbar spine model $^{8}$ consisting of a detailed FE model of the lumbar spinal column and a rigid pelvis model was used in this analysis. Detailed modeling procedures have been described in our previous studies. ${ }^{8,17-19}$ In Table 1, material properties and element types used for the FE model $^{8}$ are summarized. To analyze the effect of hybrid stabilization on the adjacent segment of the fusion level, the L4-5 segment was selected as a fusion level and installed with conventional titanium alloy rigid rods and polyetheretherketone cages (Fig. 1). Meshes in the vertebral body and pedicles of the L-4 and L-5 vertebrae were modified to incorporate pedicle screw insertion. The screw section of the pedicle screw was simplified as a beam element with the same bending stiffness as the actual screw, and the screw head section was reconstructed through FE modeling. Because our models were designed to simulate the biomechanical behavior of long-term effects after instrumentation, the bone-screw and bone-cage interfaces were assumed to be completely bonded via node sharing. The connection between the rod and screw models was simulated to be firmly connected.

\section{IH Model}

For the IH model, a previous biomechanical experiment showed that Coflex (Paradigm Spine), Wallis (Abbott Spine), DIAM (Medtronic Sofamor Danek), and X-Stop (St. Francis Medical Technologies) lumbar interspinous implants had a similar effect on flexibility, ${ }^{38}$ and we selected the DIAM system because it limited both flexion and extension, and showed a midvalue range of motion (ROM) between the intact and defect model. ${ }^{38}$ DIAM, an "H"-shaped silicone bumper was wrapped with a polyester sheath connected to 2 tethers, was additionally installed at the L3-4 segment. The bumper was modeled using a hyperelastic Mooney-Rivlin material; the strain energy density function $(\mathrm{W})$ was $\mathrm{W}=0.16\left(\mathrm{I}_{1}-3\right)+1.42\left(\mathrm{I}_{2}-3\right)$, where $I_{1}$ and $I_{2}$ are the first and second invariants of the deviatoric strain tensor, respectively. The spring element was selected for the 2 ligatured tethers and an initial tension force of $120 \mathrm{~N}$ was applied for secure tightening. The interspinous ligament in the L3-4 segment was removed for DIAM insertion.

\section{PH Model}

For the PH model, there is no substantial difference in biomechanical effects of pedicle-based dynamic stabilization by manufacturer. ${ }^{17}$ However, NFlex (Synthes Spine) has the closest instantaneous center of rotation compared with the intact model, and showed the most similar ROM to the intact model. ${ }^{17}$ The NFlex system, a semirigid rod composed of a central titanium ring surrounded by an integrated polycarbonate urethane spacer, was installed at the L3-4 segment in this study. Sliding and contact between the titanium core and sliding ring combined with 2 polycarbonate urethane spacers were modeled using surface contact elements. The rigid titanium rod section of NFlex had a circular cross-section (6-mm diameter). Material properties were assigned to the other components of the model according to a previous study. ${ }^{17}$

\section{Loading Conditions}

A cross-type rigid bar element was attached at the superior endplate of the L-1 vertebra as a loading frame, and its center was located at two-thirds of the L-1 vertebral body from the end of the anterior surface. To impose a compressive follower load, connector elements were applied between each vertebral body center. Then, local coordinates were assigned to each connector element, which were to provide the direction of action of the follower load in accordance with the modified curvature of the spinal column. Flexion, extension, lateral bending, and axial rotational moment were applied to the L-1 vertebra via a loading frame for generating the desired rotation. With proper selection of the follower load, the L4-5 intradiscal pressure (IDP) ${ }^{39}$ and the intersegmental motion ${ }^{12,14,31,32,40}$ of the intact model were close to that in the in vivo measurement. In the analysis of biomechanical changes in the models, the hybrid loading method ${ }^{29,30}$ was applied to the intact model and the 3 instrumented models to produce the same amount of motion $\left(30^{\circ}\right.$ flexion, $15^{\circ}$ extension, $20^{\circ}$ lateral bending, and $5^{\circ}$ axial rotation). Table 2 shows the magnitude of the moment applied to each model for generation of the same amount of motion. In a given plane motion, the same follower load was applied. During loading, the sacrum was fixed in all directions. ABAQUS (ver. 6.10 , Hibbitt, Karlsson \& Sorensen Inc.) was used to perform a nonlinear structural analysis of the detailed lumbar spinal column model.

\section{Results}

The motion response characteristics of the intact model varied depending on the motion segment level, although 
TABLE 1. Material properties and element types used for FE spine model

\begin{tabular}{|c|c|c|c|}
\hline Spinal Component & Element Type & Material Constants & Area $\left(\mathrm{mm}^{2}\right)$ \\
\hline \multicolumn{4}{|l|}{ Vertebra* } \\
\hline Cortical bone & S4R & $E=12,000, v=0.3$ & \\
\hline Cancellous bone & C3D8 & $E=100, v=0.2$ & \\
\hline Posterior bone & C3D8 & $E=3500, v=0.25$ & \\
\hline Endplate & S4 & $E=12,000, v=0.3$ & \\
\hline Cartilage & C3D8 & $E=75, v=0.4$ & \\
\hline \multicolumn{4}{|l|}{ Intervertebral disc $\dagger$} \\
\hline Nucleus & F3D4 & $K=2200, P_{0}=0.174$ & \\
\hline Annulus ground matrix & $\mathrm{C} 3 \mathrm{D} 8 \mathrm{H}$ & $C_{1}=0.56, C_{2}=0.14$ & \\
\hline Annulus fiber & T3D2 & & \\
\hline Anterior outermost layer & & $60(\varepsilon<0.037), 170(0.037<\varepsilon<0.058), 620(\varepsilon>0.058)$ & \\
\hline Anterior 2nd layer & & $54.75(\varepsilon<0.032), 155.125(0.032<\varepsilon<0.051), 565.75(\varepsilon>0.051)$ & \\
\hline Anterior 3rd layer & & $49.5(\varepsilon<0.026), 140.25(0.026<\varepsilon<0.045), 511.5(\varepsilon>0.045)$ & \\
\hline Anterior 4th layer & & $44.25(\varepsilon<0.021), 425.375(\varepsilon>0.021)$ & \\
\hline Anterior innermost layer & & $39(\varepsilon<0.015), 110.5(\varepsilon>0.015)$ & \\
\hline Lateral outermost layer & & $65(\varepsilon<0.018), 155(0.018<\varepsilon<0.029), 555(\varepsilon>0.029)$ & \\
\hline Lateral 2nd layer & & $59.31(\varepsilon<0.016), 141.44(0.016<\varepsilon<0.026), 506.44(\varepsilon>0.026)$ & \\
\hline Lateral 3rd layer & & $53.63(\varepsilon<0.013), 127.88(0.013<\varepsilon<0.022), 457.88(\varepsilon>0.022)$ & \\
\hline Lateral 4th layer & & $47.94(\varepsilon<0.01), 114.31(\varepsilon>0.01)$ & \\
\hline Lateral innermost layer & & $42.25(\varepsilon<0.01), 100.75(\varepsilon>0.01)$ & \\
\hline Posterior outermost layer & & $70(\varepsilon<0.05), 140(0.05<\varepsilon<0.085), 490(\varepsilon>0.085)$ & \\
\hline Posterior 2nd layer & & $63.88(\varepsilon<0.043), 127.75(0.043<\varepsilon<0.075), 447.13(\varepsilon>0.075)$ & \\
\hline Posterior 3rd layer & & $57.75(\varepsilon<0.037), 115.5(0.037<\varepsilon<0.066), 404.25(\varepsilon>0.066)$ & \\
\hline Posterior 4th layer & & $51.63(\varepsilon<0.03), 103.25(\varepsilon>0.03)$ & \\
\hline Posterior innermost layer & & $45.5(\varepsilon<0.023), 91(\varepsilon>0.023)$ & \\
\hline Ligaments (MPa) & T3D2 & & \\
\hline Anterior longitudinal ligament & & $7.8(\varepsilon<0.12), 20(\varepsilon>0.12)$ & 63.7 \\
\hline Posterior longitudinal ligament & & $10(\varepsilon<0.11), 20(\varepsilon>0.11)$ & 20 \\
\hline Capsular ligament & & $7.5(\varepsilon<0.25), 32.9(\varepsilon>0.25)$ & $30 \ddagger$ \\
\hline Interspinous ligament & & $10(\varepsilon<0.11), 11.6(\varepsilon>0.11)$ & 30 \\
\hline Ligamentum flavum & & $15(\varepsilon<0.062), 19.5(\varepsilon>0.062)$ & 40 \\
\hline Supraspinous ligament & & $8(\varepsilon<0.20), 15(\varepsilon>0.20)$ & 40 \\
\hline Transverse ligament & & $10(\varepsilon<0.18), 58.7(\varepsilon>0.18)$ & $1.8 \ddagger$ \\
\hline
\end{tabular}

$\varepsilon=$ strain.

* For vertebra: $E$ = Young's modulus (MPa); $v$ = Poisson's ratio.

† For intervertebral disc: $\mathrm{K}=$ bulk modulus $(\mathrm{MPa}) ; \mathrm{PO}=$ initial pressure $(\mathrm{MPa})$; the strain energy density function is $\mathrm{W}=\mathrm{C}_{1}\left(\mathrm{I}_{1}-3\right)+\mathrm{C}_{2}\left(\mathrm{I}_{2}-3\right)$, where $\mathrm{I}_{1}$ and $\mathrm{I}_{2}$ are first and second invariants of the deviatoric strain tensor, respectively, and $\mathrm{C}_{1}$ and $\mathrm{C}_{2}$ are constants that express the material properties.

$\ddagger$ On each side.

the motion at each level was in good agreement with the in vivo measurements. The simulation results for IDP at the intact L4-5 segment also matched the in vivo measurements. ${ }^{39}$ After rigid fixation at the L4-5 level and additional instrumentation at the transition segment (L3-4), the kinematic and mechanical compensation of the instrumented segments was distributed among other segments. Figure 2 shows the intersegmental rotation before and after instrumentation in 4 different loading directions. Compared with the intact case, fusion produced an $18.8 \%$, $9.3 \%, 11.7 \%$, and $13.7 \%$ increase in motion at the L3-4 segment under flexion, extension, lateral bending, and axial rotational loading, respectively. The ROM increment at this segment was reduced with additional instrumentation at this level. The IH model using the DIAM system showed $8.4 \%,-33.9 \%, 6.9 \%$, and $2.0 \%$ changes in motion compared with the normal model, whereas the change in motion was $-30.4 \%,-26.7 \%,-23.0 \%$, and $12.9 \%$ after instrumentation with the NFlex device.

According to the motion compensation after hybrid stabilization, the $\mathrm{PH}$ model showed larger ROMs at the adjacent segment (L2-3) to the transition level than the $\mathrm{IH}$ model in all loading directions, and motion increases of $14.3 \%, 3.4 \%, 15.0 \%$, and $0.8 \%$ were calculated compared with the motion in the IH model under flexion, extension, lateral bending, and axial rotational loading, respectively. 


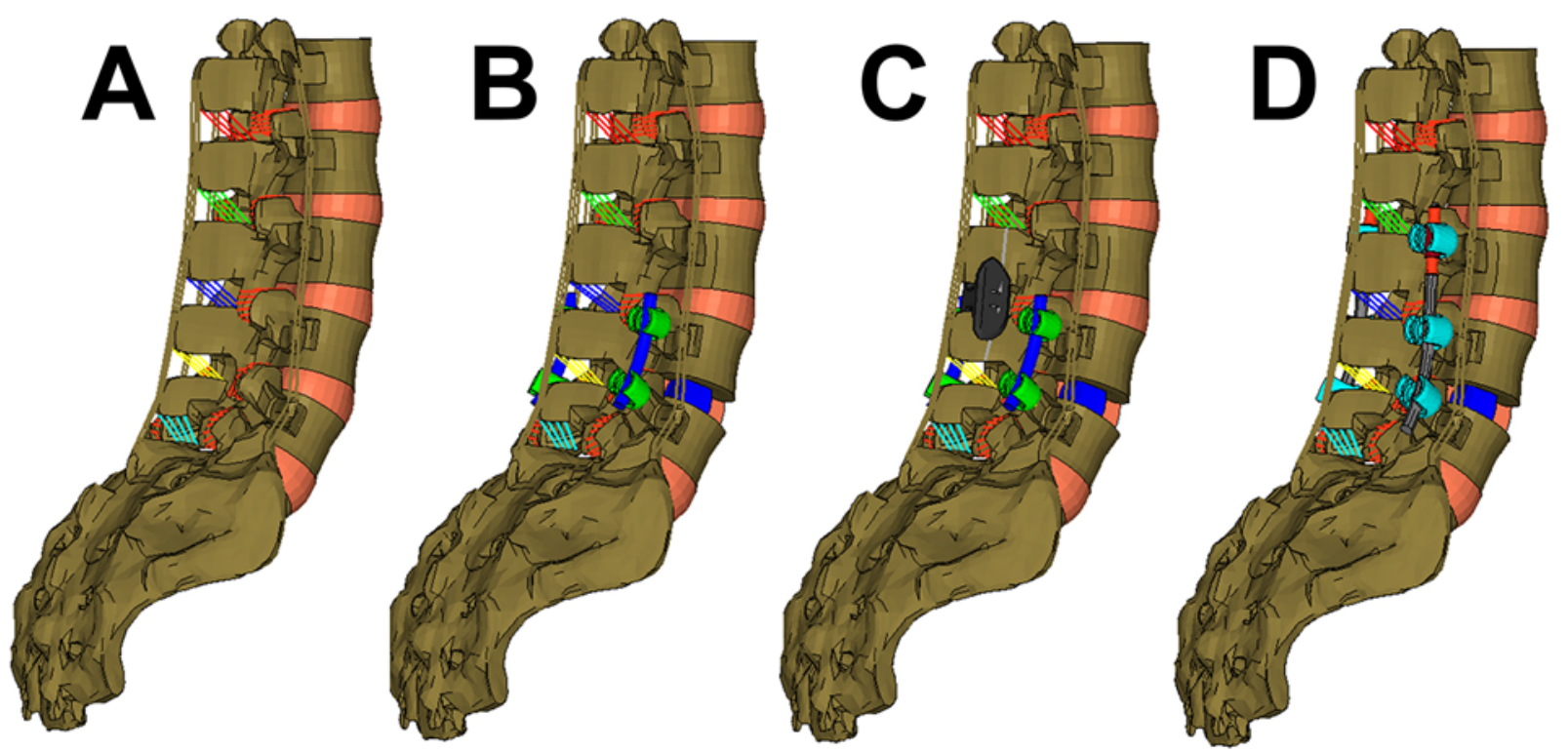

FIG. 1. Images showing the developed models: intact lumbosacral spine model for an adult (A), rigid fusion model (B), hybrid stabilization model using DIAM (C), and hybrid stabilization model using NFlex (D). For the fusion model, a conventional rigid titaniumalloy rod and cage were installed on the L4-5 segment. For the hybrid stabilization model, an interspinous device (DIAM) or PDS system (NFlex) were additionally placed on the L3-4 segment of the rigid fusion model. In the instrumented models, some part of the annulus was removed for clear visibility of the cage model. Figure is available in color online only.

The IH and PH models showed decreased IDP at the transition segment compared with the fusion model; however, the IDP at the adjacent segment (L2-3) increased in all loading directions except under extension (Fig. 3). The PH model showed the highest IDP at this level in all loading directions except under extension.

\section{Discussion}

Ideal hybrid stabilization has to preserve normal ROM as much as possible, and the transition segment has to not be too rigid or movable to preserve both the transition and adjacent segments, because a rigid transition segment transfers excessive stress to the adjacent segments from the transition segment. ${ }^{6,10}$ At the transition segment, both the IH and PH models suppress the kinematic compensation from the fusion segment and demonstrate smaller ROM and IDP than the fusion model in all loading directions. These values for the $\mathrm{PH}$ model were even lower than those for the intact model in all loading directions except under axial rotation, and the IH model showed smaller ROM and IDP than the intact model, especially under extension, because the ISD is designed to distract the foramen. It showed that the PH model appears to excessively restrict ROM at the transition segment, whereas the IH model may adequately control ROM. A previous biomechanical simulation study found that approximately $41 \%$ of the normal ROM was lost after NFlex placement. ${ }^{17}$

Clinical studies have reported that unintended fusion or implant failure commonly occurs in patients who undergo PDS. ${ }^{21,35,36}$ The PDS devices such as NFlex and Dynesys (Zimmer) are approved by the FDA only as fusion devices and not as motion-preservation devices. ${ }^{21,36}$ Putzier et al. reported that the hybrid use of rigid and dynamic fixation such as Dynesys increased problems associated with device failure and induced ASD progression at the superior segment. ${ }^{34}$ However, several studies reported that the DIAM system might compensate for the instability appropriately except under extension. . $^{20,25,33,38}$

Because of restriction of ROM and IDP of hybrid sta-

TABLE 2. Rotational moments $(\mathrm{Nm})$ required in stabilized models to achieve overall ROM equal to the intact model, and follower load for producing similar in vivo IDP at the L4-5 level

\begin{tabular}{lrrrrr}
\hline & & & \multicolumn{2}{c}{ Hybrid Stabilization } & \multicolumn{2}{c}{ Follower } \\
\cline { 4 - 5 } Loading & Intact & Fusion & IH & PH & Load (N) \\
\hline $30^{\circ}$ flexion & 12.0 & 18.4 & 19.6 & 23.0 & 1300 \\
\hline $15^{\circ}$ extension & 6.6 & 8.5 & 9.5 & 9.2 & 1000 \\
\hline $20^{\circ}$ lateral bending & 10.5 & 12.1 & 12.3 & 14.6 & 800 \\
\hline $5^{\circ}$ axial rotation & 4.8 & 5.6 & 5.8 & 5.8 & 800 \\
\hline Erect standing & - & - & - & - & 800 \\
\hline
\end{tabular}

Values are $\mathrm{Nm}$ unless otherwise specified. 

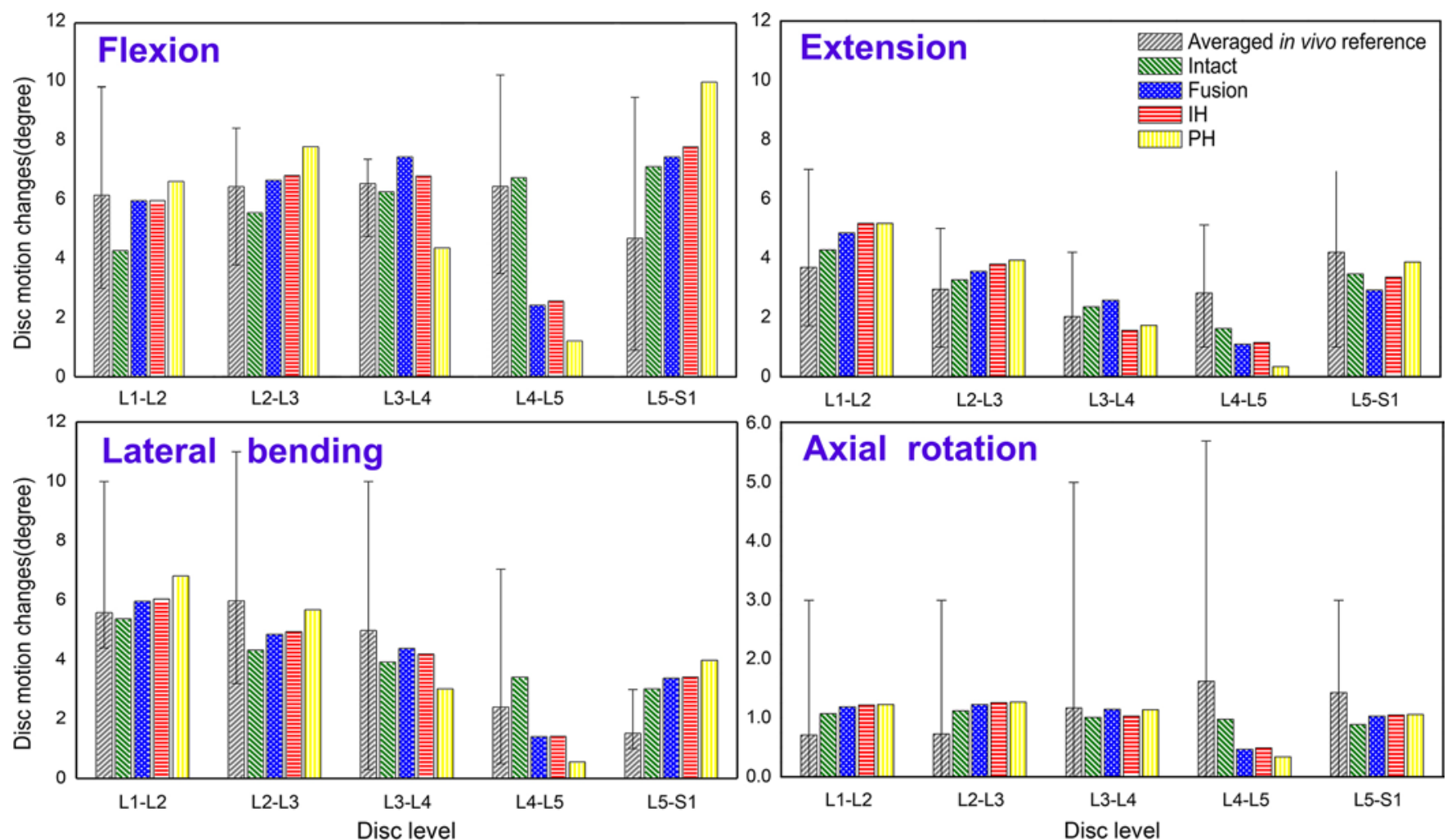

FIG. 2. Comparison of the intersegmental ROM among the intact model, fusion, $I H$, and $P H$. Results for the intact case were compared with the average in vivo measurement values from previous studies.12,14,31,32,39,40 The ranges represent the maximum and minimum values of different volunteers. Figure is available in color online only.
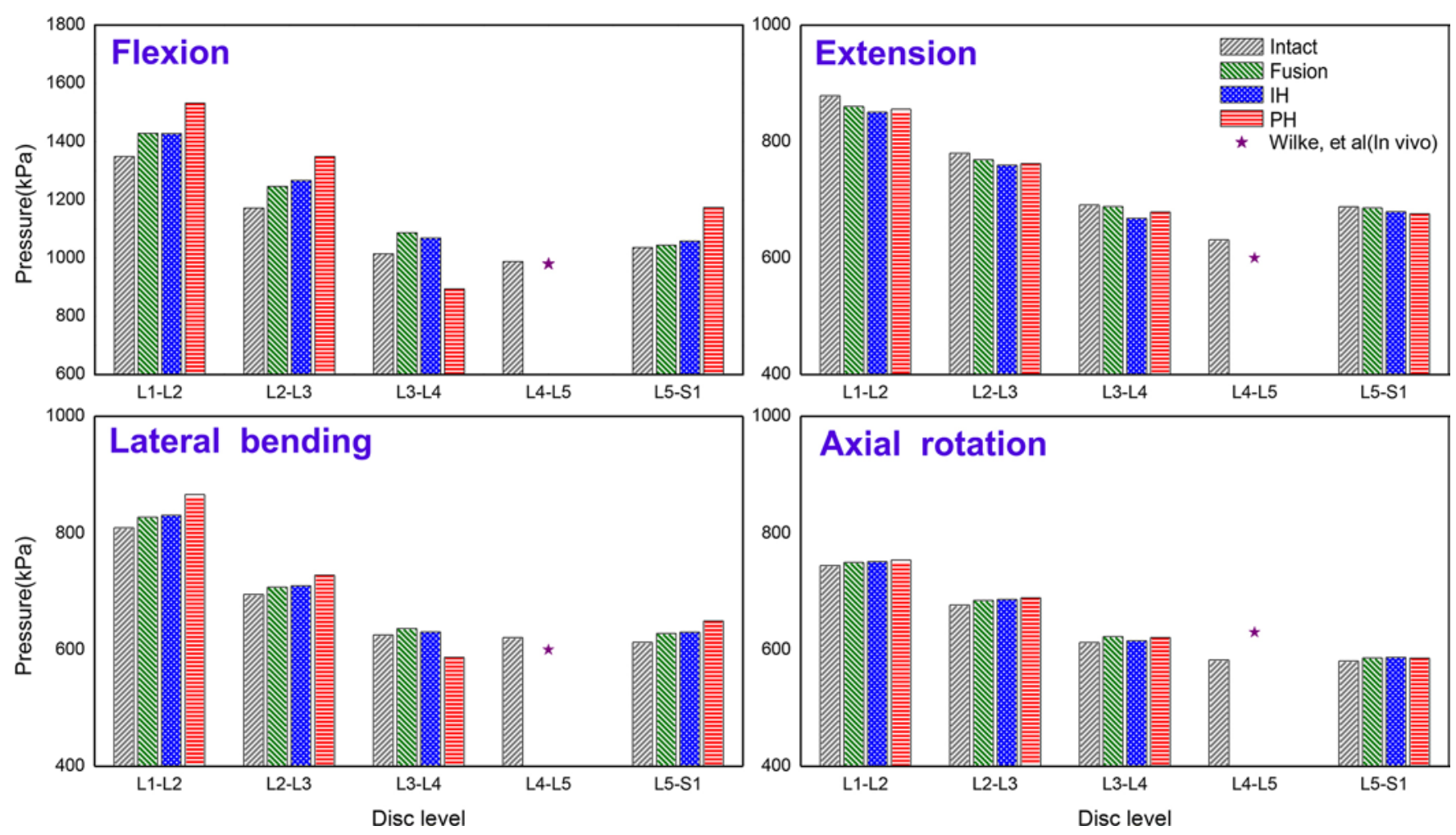

FIG. 3. IDPs at each segment of the intact model, fusion, IH, and PH. The IDP at the L4-5 segment was compared with in vivo measurements. Wilke et al. refers to Wilke et al., 2001. ${ }^{39}$ Figure is available in color online only. 
bilization models at the transition segment, ROM and IDP at the adjacent segment (L2-3) to the transition level of the hybrid models might be greater than those of the fusion model, and those of the PH might be higher than those of the IH model. The stiffness difference at the transition segment between the IH and PH models seemed to produce these changes. A previous clinical study reported that one of the main proposed benefits of dynamic stabilization is a decrease in ASD; however, clinical data supporting this claim regarding PDS are unclear. ${ }^{35}$ Other studies have concluded that PDS at the adjacent segment was not recommended in patients with clinically asymptomatic ASD. ${ }^{6,34}$ A previous meta-analysis also addressed the issue that PDS has no competitive advantage for ASD prevention compared with fusion surgery. ${ }^{21}$ However, clinical studies addressing $\mathrm{IH}$ demonstrated that $\mathrm{IH}$ achieved significantly lower ASD than conventional fusion surgery. ${ }^{20,25}$

The result of this FE study indicates that the ISD is more suitable for the transition segment of hybrid stabilization. Moreover, IH has some advantages compared with PDS at the transition segment. ${ }^{6,10}$ Installing pedicle screws at the transition segment during PH could cause facet joint damage and requires a long incision and paraspinal muscle dissection, which could be avoided in ISD placement. ISD installation usually takes less than 30 minutes, and minimal dissection of the posterior elements is required. ${ }^{1,20}$

This FE study has certain limitations like any other computational modeling investigation. We have applied the same follower load derived for the intact case to the stabilized cases. In the in vivo state, to create the same posture as an intact case when stabilization was to be applied at a certain level of the spine, some changes in the activation of the deep muscle could have been generated that would have led to changes in the follower load. However, we consistently applied a follower load with the same magnitude and direction.

We used the hybrid loading method suggested by Panjabi et al.,"29 which was designed to represent the actual scenario of the biomechanical evaluation of the adjacent spinal level following surgical procedures and implantation. ${ }^{13,29}$ The hybrid loading method used in this analysis eventually generated the same amount of motion for 4 different models despite stiffness changes at the stabilized segment. However, it is expected that the change in spinal stiffness would induce a different motion ratio between the lumbar spine and lower extremity to produce the same trunk motion in vivo. According to the previous in vivo measurement, ${ }^{24}$ the motion-lost degenerated disc at the L4-5 level showed significantly less whole lumbar motion. After lumbar spinal fusion, patients exhibited decreased muscle activity and reaching distance during the forward reaching movement compared with the healthy controls. During the forward reaching movement, the patients tended to use a muscle strategy that relied more on leg muscles and less on the lumbar extensor muscles. ${ }^{7,19}$ These findings indicate that "the trade-off effect" of the intersegmental motion change among different levels would be much less in the in vivo status.

In addition to these limitations, the morphology of the lumbosacral model was originally developed based on a young healthy adult, although most patients who receive hybrid stabilization tend to be older..$^{10}$ In addition, because of limited data sources, the degenerative changes due to osteophytes, endplate sclerosis, and annular tears were not included in this study. ${ }^{3,26}$ Models used in this study only assessed biomechanical change of the transition (L3-4) and adjacent segments (L2-3) with 2 different instrumentation devices. Actually, ASD would develop from multifactorial causes and this study addressed only some of them. Among various hybrid stabilization techniques based on ISD and PDS, we selected DIAM and NFlex, respectively, as representative devices of each group. Therefore, these results need to be interpreted with caution before generalizing to all $\mathrm{IH}$ and $\mathrm{PH}$.

\section{Conclusions}

Lumbar hybrid stabilization using both ISD and PDS decreases the fusion-induced excessive motion at the transition segment. At the segment adjacent to the transition level, the PH model induced higher stress than the IH model under the hybrid loading condition. Such a difference may eventually influence the likelihood of ASD. Detailed clinical studies will be required to examine our findings according to the type of stabilization device.

\section{Acknowledgments}

This research was supported by the Basic Science Research Program through the National Research Foundation of Korea (NRF) funded by the Ministry of Science, ICT \& Future Planning (grant no. 2015R1A2A2A01008329).

\section{References}

1. Arnold PM, Friis EA: Editorial: Biomechanical effects of interspinous process devices using a hybrid testing protocol. J Neurosurg Spine 23:197-199, 2015

2. Baioni A, Di Silvestre M, Greggi T, Vommaro F, Lolli F, Scarale A: Does hybrid fixation prevent junctional disease after posterior fusion for degenerative lumbar disorders? A minimum 5-year follow-up study. Eur Spine J 24 (Suppl 7):855-864, 2015

3. Benneker LM, Heini PF, Anderson SE, Alini M, Ito K: Correlation of radiographic and MRI parameters to morphological and biochemical assessment of intervertebral disc degeneration. Eur Spine J 14:27-35, 2005

4. Cheh G, Bridwell KH, Lenke LG, Buchowski JM, Daubs MD, Kim Y, et al: Adjacent segment disease followinglumbar/thoracolumbar fusion with pedicle screw instrumentation: a minimum 5-year follow-up. Spine (Phila Pa 1976) 32:2253-2257, 2007

5. Chen WJ, Lai PL, Tai CL, Chen LH, Niu CC: The effect of sagittal alignment on adjacent joint mobility after lumbar instrumentation - a biomechanical study of lumbar vertebrae in a porcine model. Clin Biomech (Bristol, Avon) 19:763768, 2004

6. Chien CY, Kuo YJ, Lin SC, Chuang WH, Luh YP: Kinematic and mechanical comparisons of lumbar hybrid fixation using Dynesys and Cosmic systems. Spine (Phila Pa 1976) 39:E878-E884, 2014

7. Choi HW, Kim YE: Contribution of paraspinal muscle and passive elements of the spine to the mechanical stability of the lumbar spine. Int J Precis Eng Manuf 13:993-1002, 2012

8. Choi HW, Kim YE, Chae SW: Effects of the level of monosegmental dynamic stabilization on the whole lumbar spine. Int J Precis Eng Manuf 17:603-611, 2016 
9. Chou WY, Hsu CJ, Chang WN, Wong CY: Adjacent segment degeneration after lumbar spinal posterolateral fusion with instrumentation in elderly patients. Arch Orthop Trauma Surg 122:39-43, 2002

10. Chuang WH, Lin SC, Chen SH, Wang CW, Tsai WC, Chen YJ, et al: Biomechanical effects of disc degeneration and hybrid fixation on the transition and adjacent lumbar segments: tradeoff between junctional problem, motion preservation, and load protection. Spine (Phila Pa 1976) 37:E1488-E1497, 2012

11. Coe JD, Kitchel SH, Meisel HJ, Wingo CH, Lee SE, Jahng TA: NFlex dynamic stabilization system: two-year clinical outcomes of multi-center study. J Korean Neurosurg Soc 51:343-349, 2012

12. Dvorák J, Panjabi MM, Chang DG, Theiler R, Grob D: Functional radiographic diagnosis of the lumbar spine. Flexion-extension and lateral bending. Spine (Phila Pa 1976) 16:562-571, 1991

13. Erbulut DU, Zafarparandeh I, Hassan CR, Lazoglu I, Ozer AF: Determination of the biomechanical effect of an interspinous process device on implanted and adjacent lumbar spinal segments using a hybrid testing protocol: a finite-element study. J Neurosurg Spine 23:200-208, 2015

14. Haughton VM, Rogers B, Meyerand ME, Resnick DK: Measuring the axial rotation of lumbar vertebrae in vivo with MR imaging. AJNR Am J Neuroradiol 23:1110-1116, 2002

15. Hilibrand AS, Robbins M: Adjacent segment degeneration and adjacent segment disease: the consequences of spinal fusion? Spine J 4 (6 Suppl):190S-194S, 2004

16. Hudson WR, Gee JE, Billys JB, Castellvi AE: Hybrid dynamic stabilization with posterior spinal fusion in the lumbar spine. SAS J 5:36-43, 2011

17. Jahng TA, Kim YE, Moon KY: Comparison of the biomechanical effect of pedicle-based dynamic stabilization: a study using finite element analysis. Spine J 13:85-94, 2013

18. Kim YE, Choi HW: Effect of disc degeneration on the muscle recruitment pattern in upright posture: a computational analysis. Comput Methods Biomech Biomed Engin 18:1622-1631, 2015

19. Kim YE, Choi HW: Paraspinal muscle activation in accordance with mechanoreceptors in the intervertebral discs. Proc Inst Mech Eng H 227:138-147, 2013

20. Lee CH, Hyun SJ, Kim KJ, Jahng TA, Yoon SH, Kim HJ: The efficacy of lumbar hybrid stabilization using the DIAM to delay adjacent segment degeneration: an intervention comparison study with a minimum 2-year follow-up. Neurosurgery 73 (2 Suppl Operative):ons224-ons232, 2013

21. Lee CH, Jahng TA, Hyun SJ, Kim CH, Park SB, Kim KJ, et al: Dynamic stabilization using the Dynesys system versus posterior lumbar interbody fusion for the treatment of degenerative lumbar spinal disease: a clinical and radiological outcomes-based meta-analysis. Neurosurg Focus 40(1):E7, 2016

22. Lee CK, Langrana NA: Lumbosacral spinal fusion. A biomechanical study. Spine (Phila Pa 1976) 9:574-581, 1984

23. Lee SE, Jahng TA, Kim HJ: Hybrid surgery combined with dynamic stabilization system and fusion for the multilevel degenerative disease of the lumbosacral spine. Int J Spine Surg 9:45, 2015

24. Lee SH, Daffner SD, Wang JC, Davis BC, Alanay A, Kim JS: The change of whole lumbar segmental motion according to the mobility of degenerated disc in the lower lumbar spine: a kinetic MRI study. Eur Spine J 24:1893-1900, 2015

25. Lu K, Liliang PC, Wang HK, Liang CL, Chen JS, Chen $\mathrm{TB}$, et al: Reduction in adjacent-segment degeneration after multilevel posterior lumbar interbody fusion with proximal DIAM implantation. J Neurosurg Spine 23:190-196, 2015

26. Lu YM, Hutton WC, Gharpuray VM: Can variations in intervertebral disc height affect the mechanical function of the disc? Spine (Phila Pa 1976) 21:2208-2217, 1996
27. Lund T, Oxland TR: Adjacent level disk disease-is it really a fusion disease? Orthop Clin North Am 42:529-541, viii, 2011

28. Malakoutian M, Volkheimer D, Street J, Dvorak MF, Wilke HJ, Oxland TR: Do in vivo kinematic studies provide insight into adjacent segment degeneration? A qualitative systematic literature review. Eur Spine J 24:1865-1881, 2015

29. Panjabi M, Henderson G, Abjornson C, Yue J: Multidirectional testing of one- and two-level ProDisc-L versus simulated fusions. Spine (Phila Pa 1976) 32:1311-1319, 2007

30. Panjabi MM: Hybrid multidirectional test method to evaluate spinal adjacent-level effects. Clin Biomech (Bristol, Avon) 22:257-265, 2007

31. Pearcy M, Portek I, Shepherd J: Three-dimensional x-ray analysis of normal movement in the lumbar spine. Spine (Phila Pa 1976) 9:294-297, 1984

32. Pearcy MJ, Tibrewal SB: Axial rotation and lateral bending in the normal lumbar spine measured by three-dimensional radiography. Spine (Phila Pa 1976) 9:582-587, 1984

33. Phillips FM, Voronov LI, Gaitanis IN, Carandang G, Havey RM, Patwardhan AG: Biomechanics of posterior dynamic stabilizing device (DIAM) after facetectomy and discectomy. Spine J 6:714-722, 2006

34. Putzier M, Hoff E, Tohtz S, Gross C, Perka C, Strube P: Dynamic stabilization adjacent to single-level fusion: part II. No clinical benefit for asymptomatic, initially degenerated adjacent segments after 6 years follow-up. Eur Spine J 19:2181-2189, 2010

35. Schroeder GD, Murray MR, Hsu WK: A review of dynamic stabilization in the lumbar spine. Oper Tech Orthop 21:235-239, 2011

36. Sengupta DK, Herkowitz HN: Pedicle screw-based posterior dynamic stabilization: literature review. Adv Orthop 2012:424268, 2012

37. Volkheimer D, Malakoutian M, Oxland TR, Wilke HJ: Limitations of current in vitro test protocols for investigation of instrumented adjacent segment biomechanics: critical analysis of the literature. Eur Spine J 24:1882-1892, 2015

38. Wilke HJ, Drumm J, Häussler K, Mack C, Steudel WI, Kettler A: Biomechanical effect of different lumbar interspinous implants on flexibility and intradiscal pressure. Eur Spine J 17:1049-1056, 2008

39. Wilke H, Neef P, Hinz B, Seidel H, Claes L: Intradiscal pressure together with anthropometric data-a data set for the validation of models. Clin Biomech (Bristol, Avon) 16 (Suppl 1):S111-S126, 2001

40. Wong KW, Luk KD, Leong JC, Wong SF, Wong KK: Continuous dynamic spinal motion analysis. Spine (Phila Pa 1976) 31:414-419, 2006

\section{Disclosures}

The authors report no conflict of interest concerning the materials or methods used in this study or the findings specified in this paper.

\section{Author Contributions}

Conception and design: CH Lee. Analysis and interpretation of data: YE Kim, HJ Lee. Drafting the article: YE Kim, CH Lee. Critically revising the article: DG Kim, CH Kim. Reviewed submitted version of manuscript: all authors. Approved the final version of the manuscript on behalf of all authors: YE Kim. Study supervision: YE Kim, HJ Lee, CH Kim.

\section{Correspondence}

Young Eun Kim, Department of Mechanical Engineering, Dankook University, 152, Jukjeon-ro, Suji-gu, Yongin, Gyeonggido 16891, Korea. email: yekim@dankook.ac.kr. 